\title{
An 11-year analysis of microbial keratitis in the South West of England using brain-heart infusion broth
}

\author{
Shokufeh Tavassoli ${ }^{1} \cdot$ Gayathri Nayar $^{2} \cdot$ Kieren Darcy $^{1} \cdot$ Mariusz Grzeda $^{1} \cdot$ Jon Luck $^{3} \cdot$ O. Martin Williams ${ }^{2}$. \\ Derek Tole ${ }^{1}$
}

Received: 27 November 2018 / Revised: 25 March 2019 / Accepted: 22 April 2019 / Published online: 9 May 2019

(c) The Royal College of Ophthalmologists 2019

\begin{abstract}
Background The aims of this study were to identify the organisms responsible for microbial keratitis, as identified by corneal scrape using brain-heart infusion broth, trends over time and antimicrobial sensitivities, over an 11-year period at two eye units in the South West of England; Bristol Eye Hospital and Royal United Hospital, Bath.

Methods All corneal scrapes performed and sent for microbiological analysis between 4th April 2006 and 31st October 2017 at the two eye units were retrospectively reviewed. First-line treatment was monotherapy with levofloxacin $0.5 \%$ and second-line treatment was a combination of cefuroxime $5 \%$ and gentamicin $1.5 \%$. Both direct and enrichment cultures were used.

Results In total, 2614 corneal scrapes from 2116 patients (1082 female, mean age $47.7 \pm 21.2$ years) were identified. $38.1 \%$ $(n=996)$ were culture positive and 1195 organisms were cultured. In all, $91.6 \%$ were bacteria $(69.4 \%$ were gram-positive, $30.6 \%$ gram-negative). Coagulase-negative Staphylococci (CoNS) were the most commonly cultured organism $(n=430)$. Pseudomonas aeruginosa was the most commonly identified gram-negative organism $(n=189)$. In total, 6.9\% $(n=83)$ of organisms cultured were fungi. In all, $1.4 \%(n=17)$ were acanthamoeba. There was no statistically significant trend in the organisms observed over the study period. Sensitivity testing confirmed reasonable sensitivity to the empiric antibiotics used in clinical practice.

Conclusions This is the first report on microbial keratitis trends in the South West of England. Virulent organisms were likely to be detected on direct culture, whereas low virulent organisms such as CoNS were more likely to be detected on enrichment alone. Antibiotic sensitivity testing confirmed fluoroquinolone monotherapy as appropriate first-line treatment.
\end{abstract}

\section{Introduction}

Microbial keratitis is a potentially sight-threatening corneal condition, most commonly affecting contact lens wearers. In addition, patients with ocular surface diseases are at risk. These include keratoconjunctivitis sicca, neurotrophic keratopathy, surface disease and extended exposure to topical

Shokufeh Tavassoli

shokufeh_tavassoli@hotmail.com

1 Bristol Eye Hospital, Cornea and Refractive Surgery, Bristol, United Kingdom

2 University Hospitals Bristol and Public Health England Microbiology Laboratory Services Bristol, Bristol, United Kingdom

3 Royal United Hospital Bath, Bristol, United Kingdom steroids, following anterior segment surgical procedures, for example, corneal transplantation. It is a significant cause of visual loss worldwide [1]. Early diagnosis and treatment with topical antimicrobial therapy is essential for better recovery and visual prognosis [2]. Although empirical treatment is often initiated, corneal scrapes are routinely performed to help tailor the appropriate antimicrobial agent to the causal organism. This information provides additional value in helping to identify the most common causal organism regionally, which may vary geographically.

Recent studies have considered trends in microbial keratitis in specific areas within the UK, including Sunderland [3], Manchester [4] and Oxford [5]. A trend towards an increase in moraxella keratitis was observed over recent years [3, 4], with variation in reports of the trend in grampositive organisms (increasing [3], decreasing [4]). Culture plates (blood agar plate, chocolate agar plate, Sabouraud's agar plate and non-nutrient agar seeded with Escherichia 
coli for acanthamoeba) were used to perform the corneal scrape in these studies. As previously reported [6], brain-heart infusion (BHI) broth may provide a more pragmatic and equally efficacious method to perform corneal scrapes. We were interested to analyse the microbial keratitis trends in the South West of England, where BHI is used in all corneal scrapes, rather than culture plates, to see if similarities exist in the trends seen across the UK.

The aims of this study were thus to identify the organisms responsible for microbial keratitis, as identified by corneal scrape using $\mathrm{BHI}$, the trends over time and the antimicrobial sensitivities, over an 11-year period at two eye units in the South West of the England; Bristol Eye Hospital and Royal United Hospital, Bath.

\section{Methods}

All corneal scrape samples performed between 4th April 2006 and 31st October 2017, at the Royal United Hospital, Bath and the Bristol Eye Hospital, a tertiary referral unit, which were sent for microbiological analysis at the Public Health England Microbiology Laboratory Services Bristol were included in this study. Data collected included the age and gender of the patient, the date of the scrape, the isolates and, where appropriate, the antimicrobial sensitivities. This data were retrospectively extracted from the microbiology electronic databases.

In both units, a corneal scrape was typically performed where the corneal ulcer and infiltrate were larger than 0.5 $\mathrm{mm}$ or atypical and not improving on treatment. This was either in the eye casualty at presentation or in clinic and the decision to perform a corneal scrape was as per the judgement of the ophthalmologist. Corneal samples were taken using a sterile scalpel blade. The samples were placed on slides for Gram staining. Further samples were taken using new scalpel blades and immediately placed in $1 \mathrm{~mL}$ BHI broth in a sealed glass bottle, similar to the technique previously described [6]. The samples were transported inside a CE marked leak proof container.

Microbiological analysis of the samples consisted of Gram staining of the slide and culture of the BHI sample. Approximately $40 \mu \mathrm{L}$ of fluid from the BHI broth containing the sample was inoculated on to agar plates using standard laboratory methods, including chocolate agar (incubated at $35-37^{\circ} \mathrm{C}$ in $5-10 \%$ carbon dioxide), fastidious anaerobe agar (FAA) (incubated at $35-37^{\circ} \mathrm{C}$ anaerobically) and Sabouraud dextrose agar (incubated at 28-30 ${ }^{\circ} \mathrm{C}$ in air). All plates were incubated for $40-48 \mathrm{~h}$, except if the Gram stain showed branching gram-positive bacilli, the incubation time of the anaerobic FAA plates was extended to 10 days to facilitate the growth of Actinomyces species. If indicated by the clinical details (for example, the use of contact lenses, corneal ulceration or at clinician's request), corneal scrapings were inoculated onto non-nutrient agar with a lawn of Escherichia coli for culture of acanthamoeba species. Of note, where there was a clinical suspicion of acanthamoeba keratitis (AK), corneal scrape samples were separately sent for PCR to a reference laboratory in Glasgow.

For the bacteria, the difference between organisms identified on direct or enrichment culture was considered. The total numbers of the organisms included a combination of those identified by both direct and enrichment culture. For direct culture, this included the organisms that were grown on the initial plates. For enrichment culture, the BHI was incubated for a further 5 days.

In vitro antibiotic sensitivity testing was performed by the disc diffusion method or using the automated VITEK2 system (BioMeriux).

In order to assess trends over time, the data set was separated into two groups; 4th April 2006 to 31st December 2011 and 1st January 2012 to 31 st October 2017. Statistical significance between these groups was assessed using the Chi-squared test. A $p$ value of $<0.01$ was taken as statistically significant.

\section{Results}

\section{Types of organisms}

Over the 11-year study period, a total of 2614 corneal scrapes were received from 2116 patients (1082 female and 1034 male). The mean age of the patients was 47.7 years \pm 21.2 (mean \pm SD). Of the 2614 corneal scrapes, 996 were culture positive $(38.1 \%)$. There was an increase in both the numbers of corneal scrapes performed and the numbers of organisms identified with time (Table 1). The percentage of culture-positive scrapes, however, was similar between the two time frames (Table 1). A total of 1195 organisms were cultured, including samples that cultured multiple organisms. Of the 1195 organisms isolated, 91.6\% $(n=1095)$ were bacteria, $3.2 \%(n=38)$ were yeasts, $3.8 \%(n=45)$ were moulds and $1.4 \%(n=17)$ were acanthamoeba species (see Fig. 1). Of the 1095 bacterial species cultured during the study period, $69.4 \%$ ( $n=760$, with 350 isolated on direct culture) were gram-positive and $30.6 \%(n=335$, with 270 isolated on direct culture) were gram-negative. Figure 2 summarises the trend in the types of organisms identified on scrape over time. As seen there was an overall trend towards an increase in the percentage of grampositive bacteria with time and a trend towards a decrease in the percentage of gram-negative bacteria with time, these, however, did not reach statistical significance (Table 1). 
Table 1 Summary of microbiological profile of scrapes carried out between 4th April 2006 and 31st October 2017 at Bristol Eye Hospital and Royal United Hospital, Bath, separated according to years to assess for trends over time

\begin{tabular}{|c|c|c|c|}
\hline & $\begin{array}{l}2006-2011 \\
\text { (Total } \\
\text { organisms } \\
N=550 \text { ) }\end{array}$ & $\begin{array}{l}2012-2017 \\
\text { (Total } \\
\text { organisms } \\
N=645 \text { ) }\end{array}$ & $P$ value* \\
\hline Total scrapes & 1187 & 1427 & \\
\hline Total positive scrapes ${ }^{\mathrm{a}}$ & 454 & 542 & 0.89 \\
\hline $\begin{array}{l}\text { Percentage culture } \\
\text { positive }\end{array}$ & $38.24 \%$ & $37.98 \%$ & 0.89 \\
\hline Gram-positive total & 339 & 421 & 0.193 \\
\hline Streptococci & 39 & 45 & 0.9387 \\
\hline S. aureus & 33 & 48 & 0.323 \\
\hline CoNS & 193 & 237 & 0.5528 \\
\hline Bacilli & 20 & 20 & 0.6079 \\
\hline Others $^{b}$ & 54 & 71 & 0.503 \\
\hline Gram-negative total & 165 & 170 & 0.1622 \\
\hline Pseudomonas spp. & 94 & 95 & 0.2647 \\
\hline Moraxella $\mathrm{spp}^{\mathrm{c}}$ & 15 & 22 & 0.4965 \\
\hline Others $^{\mathrm{d}}$ & 56 & 53 & 0.2397 \\
\hline Fungi & 41 & 42 & 0.5228 \\
\hline Acanthamoeba $\mathrm{spp}^{\mathrm{e}}$ & 5 & 12 & 0.1663 \\
\hline
\end{tabular}

${ }^{a}$ Note the total number of organisms is greater than the total positive scrape number, as this includes samples that cultured multiple organisms

${ }^{\mathrm{b}}$ Gram-positive other includes Enterococci, Micrococcus spp, diphtheroids ${ }^{c}$ Moraxella species include $M$. catarrhalis, M. lacunata, M. nonliquefaciens, Moraxella spp. (MOR)

${ }^{\mathrm{d}}$ Gram-negative other includes Enterobacter spp, Citrobacter spp, Escherichia spp, Haemophilus influenzae, Klebsiella spp, Morganella spp, Proteus spp and Serratia spp

${ }^{\mathrm{e}}$ Note during the study period clinically that there were a greater number of acanthamoeba keratitis (AK) cases seen. Other methods including PCR sent to a reference laboratory and confocal microscopy were used for the diagnosis of these cases and these scrape results may not therefore provide a true representation of the number of $\mathrm{AK}$ cases seen

${ }^{*} p$ value calculated using the chi-squared test. No statistically significant difference identified between the two time frames and organisms seen, or in the percentage of culture-positive scrapes

Coagulase-negative Staphylococci (CoNS) were the most frequently isolated organism (Table 1) as well as the most frequently isolated gram-positive organism $(n=430)$, of which $44.2 \%(n=190)$ were isolated on direct culture and $55.8 \%(n=240)$ were isolated on enrichment culture only. There were over seven species of CoNS cultured. It was noted that the proportion of unspeciated CoNS decreased over time and Staphylococcus epidermidis increased over time. This was likely owing to the introduction of the Matrix Assisted Laser Desorption IonisationTime of Flight Mass Spectrometry in 2012, which allowed

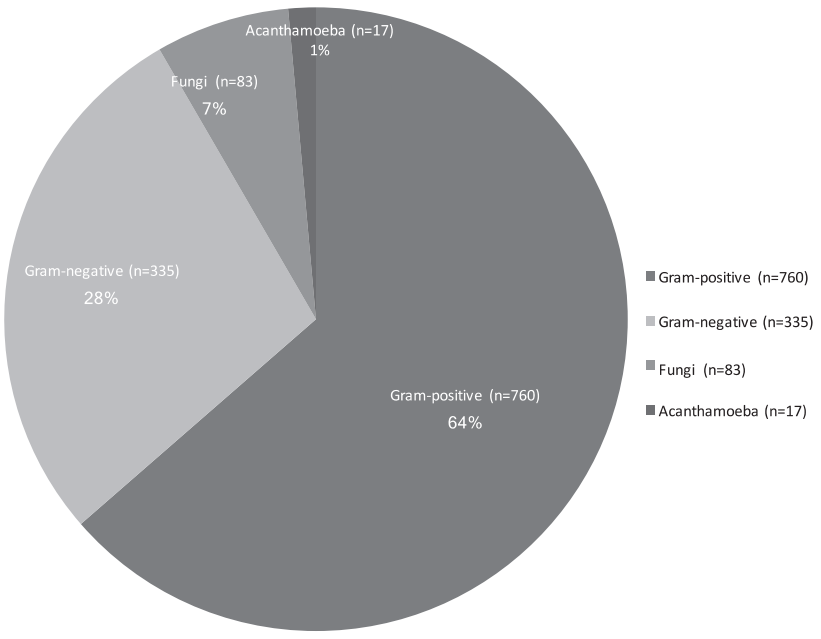

Fig. 1 Summary of the groups of organisms identified on corneal scrapes during the 11-year study period

more-accurate identification of organisms. Other grampositive organisms identified include: Staphylococcus aureus $(n=81$; direct culture $=62)$, Streptococcus pneumoniae $(n=27$; direct culture $=24)$, Virdans Streptococci $(n$ $=42$; direct culture $=20)$, diphtheroids $(n=58$; direct culture $=19)$, Micrococcus species $(n=44$; direct culture $=6$ ). The most frequently identified gram-negative organism was Pseudomonas aeruginosa $(n=189$; direct culture $=162)$, and members of the Enterobacteriaceae $(n=24$; direct culture $=20$ ). The most commonly isolated member of the Enterobacteriaceae family was Serratia marcescens. Moraxella spp were cultured in $n=37:$ M. catarrhalis $(n=$ 10 ; direct culture $=9), M$. nonliquifasciens $(n=13$; direct culture $=9$ ). There was no significant trend over the study period in the numbers of identified Pseudomonas or Moraxella species (Table 1).

Fungi were identified in $6.9 \%$ of cases $(n=83) ; 46 \%$ of which were yeasts $(n=38)$ and $54 \%$ of which were moulds $(n=45)$. As seen in Table 1, there was no trend with time in the numbers of fungi cultured. There were 17 cases of acanthamoeba identified during the 11-year study period. As seen in Table 1, the number of acanthamoeba cases increased over recent years, this however did not reach statistical significance. Of note, during the study period clinically there were a greater number of AK cases seen [7]. Other methods including PCR sent to a reference laboratory and confocal microscopy were used for the diagnosis of these cases. The scrape results in isolation therefore, do not truly represent the total number of AK cases seen clinically.

\section{Trends over time}

Table 1 shows a summary of the organisms identified over the 11-year study period. Although there was a trend towards an increase in the percentage of gram-positive 


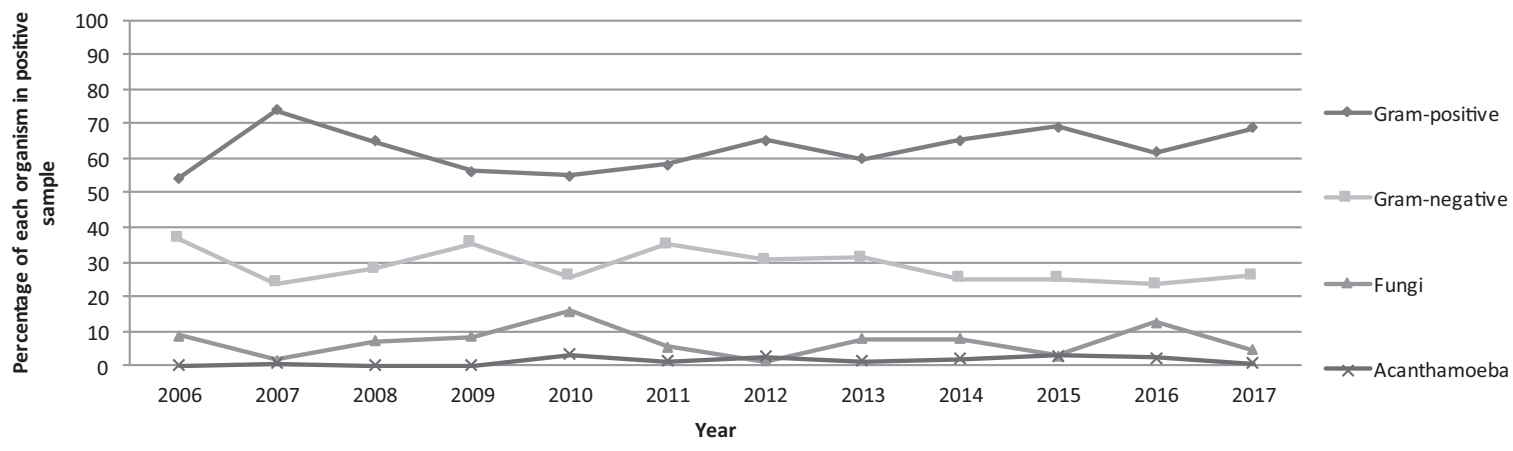

Fig. 2 Scatter plot showing percentage of each organism identified on corneal scrape over the 11-year period

Table 2 Summary of antibiotic sensitivities

\begin{tabular}{|c|c|}
\hline Organism & Antibiotic sensitivities ( $\%$ sensitive) \\
\hline Staphylococcus aureus & $\begin{array}{l}\text { Chloramphenicol }(100 \%) \\
\text { Ciprofloxacin }(91 \%) \\
\text { Fusidic acid }(86 \%) \\
\text { Gentamicin }(100 \%) \\
\text { Note } 5 \% \text { meticillin resistant (MRSA) }\end{array}$ \\
\hline Streptococcus pneumoniae & $\begin{array}{l}\text { Chloramphenicol }(100 \%) \\
\text { Levofloxacin }(100 \%) \\
\text { Penicillin }(100 \%)\end{array}$ \\
\hline Pseudomonas aeruginosa & $\begin{array}{l}\text { Chloramphenicol }(0 \%) \\
\text { Ciprofloxacin }(99.4 \%) \\
\text { Gentamicin }(99.4 \%)\end{array}$ \\
\hline Enterobacter spp & $\begin{array}{l}\text { Chloramphenicol }(90 \%) \\
\text { Ciprofloxacin }(97 \%) \\
\text { Gentamicin }(97 \%) \\
\text { Cefuroxime }(40 \%)\end{array}$ \\
\hline $\begin{array}{l}\text { Haemophilus-like organisms } \\
\text { (including Haemophilus spp, } \\
\text { Moraxellaspp. and } \\
\text { Pasteurella spp), }\end{array}$ & $\begin{array}{l}\text { Amoxicillin }(31 \%) \\
\text { Chloramphenicol }(96 \%) \\
\text { Ciprofloxacin }(100 \%) \\
\text { Gentamicin }(100 \%)\end{array}$ \\
\hline
\end{tabular}

Note the antibiotic sensitivities were not always directly tested for the antibiotics used clinically. For example, sensitivity was not tested directly for levofloxacin; the sensitivities for ciprofloxacin, also a fluoroquniolone were taken as being similar. The percentage reported in the percentage of those tested sensitive to the specific antibiotic. Percentage resistant to that particular antibiotic would therefore be 100 minus the percentage reported as sensitive in the above table

bacteria and a trend towards a decrease in the percentage of gram-negative bacteria with time (Fig. 2), this did not reach statistical significance over the study period.

\section{Antimicrobial sensitivities}

Table 2 shows a summary of antibiotic sensitivities. The antibiotic sensitivity information was not available for all the antibiotics used clinically. For example, if sensitivity was not tested directly for levofloxacin; the sensitivities for ciprofloxacin, also a fluoroquinolone, were taken as being similar. Of the fungi, only 23 had sensitivity testing, which was too small to draw any reliable conclusions from.

\section{Discussion}

This is the first study analysing the microbiology and trends in corneal scrape profiles from two units, Bristol Eye Hospital and Royal United Hospital Bath, in the South West of England, where the BHI collection method for corneal scrapes is utilised. The overall proportion of culture positive samples over 11 -years in this study was $38.1 \%$ which is comparable to that from previous reports in the UK (Table 3; 32.6\% [4], 33\% [6], 44.5\% [3]. As shown in Table 1, there was an increase in the number of corneal scrapes performed over the second time period and a corresponding increase in the numbers of organisms identified. There was no significant difference in the percentage of scrapes that were culture positive over time. The increase in the number of corneal scrapes performed over recent years may be owing to an overall increase in the numbers of microbial keratitis cases seen over the study period, as well as a lower threshold for performing scrapes, including scrapes on non-infectious cases such as marginal keratitis, which may also explain the low culture-positive rates. In the two units during the study period BHI was used, in a technique previously described, which is considered to be a more efficient way of performing corneal scrapes particularly in a busy casualty setting [6].

In our study, gram-positive bacteria were found to be the most frequently cultured group of isolates with CoNS as the most frequently identified organism. The most frequently identified gram-negative organism was Pseudomonas aeruginosa. As seen in Fig. 2 there was a trend towards a relative increase in gram-positive organisms and a relative 
Table 3 A summary of our major findings in comparison with other recent UK studies

\begin{tabular}{|c|c|c|c|c|c|c|}
\hline Study & $\begin{array}{l}\text { Number of } \\
\text { years }\end{array}$ & $\begin{array}{l}\text { Corneal } \\
\text { scrape method }\end{array}$ & $\begin{array}{l}\text { Number of } \\
\text { organisms }\end{array}$ & $\begin{array}{l}\text { Culture } \\
\text { positive rate }\end{array}$ & $\begin{array}{l}\text { Most-commonly } \\
\text { identified organism }\end{array}$ & $\begin{array}{l}\text { Statistically significant trends } \\
\text { over time }\end{array}$ \\
\hline $\begin{array}{l}\text { Our study: South } \\
\text { West England }\end{array}$ & $112016-2017$ & BHI & 1195 & $38.1 \%$ & CoNS (no trend) & No trends with time. \\
\hline Sunderland [3] & 10 2008-2017 & Culture plates & 478 & $44.5 \%$ & $\begin{array}{l}\text { CoNS with } \\
\text { increasing trend }\end{array}$ & $\begin{array}{l}\text { Increase in CoNS. Decrease in } \\
\text { pseudomonas. }\end{array}$ \\
\hline Manchester [4] & 12 2004-2015 & Culture plates & 1379 & $32.6 \%$ & $\begin{array}{l}\text { CoNS with } \\
\text { decreasing trend }\end{array}$ & $\begin{array}{l}\text { Decrease in gram-positive. } \\
\text { Increase in moraxella, fungi } \\
\text { and acanthamoeba. }\end{array}$ \\
\hline Oxford [5] & 10 1999-2009 & Culture plates & 267 & $54 \%$ & $\begin{array}{l}\text { CoNS with } \\
\text { increasing trend }\end{array}$ & Increase in CoNS. \\
\hline
\end{tabular}

decrease in gram-negative organisms over time, however, as summarised in Table 1, this did not reach statistical significance. There was an increase in the actual numbers of Moraxella and acanthamoeba cases seen with time (Table 1), though this did not reach statistical significance. There was no trend in the numbers of fungi identified in this study, although a previous study from the UK has suggested a trend towards an increase in fungal keratitis in recent years [4]. Gram-positive bacteria were also the most common isolates in other studies from the UK (Table 3; [3-5, 8]). The CoNS were also the most frequently observed organism in the Sunderland and Oxford series, with an increasing trend $[3,5]$ and Manchester series, with a decreasing trend [4]. CoNS have also been found to be the most common organisms isolated in both Indian [9] and Saudi Arabian [10] studies. In Portugal, S. aureus is reported as the most common isolate [11]. This is in contrast to other countries (Taiwan [12]; China [13]; South Florida [14]), where pseudomonas species are the most frequently identified organisms. Our data support the variations in geographic and temporal profiles of microbial keratitis [15], which further emphasises the importance of identifying emerging trends in microbial keratitis locally.

Interestingly, our results suggest a difference between the detection of bacteria on direct or enrichment culture. Organisms considered to be virulent, such as P. aeruginosa, members of the Enterobacteriaceae, $S$. aureus and $S$. pneumoniae were more likely to be detected on direct culture, rather than enrichment culture alone. In contrast, lessvirulent organisms, which may be commensals on the conjunctival surface, such as CoNS, were more likely to be detected on enrichment culture alone. Therefore, in practice clinical correlation is required to understand the significance of the microbiological result.

In vitro sensitivity testing showed reasonable susceptibility to our empiric antibiotic regimens. In vitro testing of antibiotic sensitivities is a useful guide to treatment when considered in the context of its clinical response and has in fact been shown to be predictive of the therapeutic response in bacterial keratitis [16]. However, it is important to note that the high intensity at which antibiotics are given topically in clinical practice (initially hourly day and night for 48-hours) may achieve a sufficiently high concentration to have a bactericidal effect, and therefore the actual rate of clinical sensitivity may be higher than that seen with in vitro testing. In addition, when considering resistance and susceptibility, it is important to note that these parameters are based on systemic breakpoint criteria rather than ophthalmic breakpoints [8]. This is the expected response against concentrations of the antimicrobial agent achieved in the serum, which will be different to that achieved in the cornea and aqueous humour following topical application [17]. There is very-little pharmacokinetic/pharmacodynamic data available for topical antimicrobials, which may be significantly different to systemically administered drugs [18]. Our first-line treatment is monotherapy with levofloxacin $0.5 \%$ and second-line treatment is dual therapy with cefuroxime $5 \%$ and gentamicin $1.5 \%$. As seen from our results (Table 2), the antibiotic sensitivities were not always directly tested for the antibiotics used clinically. For example, sensitivity was not tested directly for levofloxacin; the sensitivities for ciprofloxacin, also a fluoroquniolone were taken as being similar. Our data suggest reasonable activity against of the fluoroquinolones against gram-positive organisms $(91 \%$ sensitivity to ciprofloxacin for S. aureus and $100 \%$ sensitivity to levofloxacin for $S$. pneumoniae). Treatment with fluoroquinolone alone appears to be a reasonable first-line therapy for the gram-negatives $(99.4 \%$ ciprofloxacin sensitive for pseudomonas species, 97\% ciprofloxacin sensitive for enterobacteriacae, $100 \%$ ciprofloxacin sensitive for haemophilus-like organisms, including Haemophilus spp, Moraxella spp. and Pasteurella spp). In primary care, chloramphenicol is often used in cases of non-specific eye infections, and as expected, our data confirm that this is not an appropriate choice in Pseudomonal keratitis; $100 \%$ of $P$. aeruginosa isolates were resistant to chloramphenicol. Chloramphenicol remains an active agent against $S$. aureus an $S$. pneumoniae and has good activity 
against other gram-negative organisms. Of the Enterobacteriaceae, there was a relatively high rate of cefuroxime resistance (60\%), explained by the fact that most of the cefuroxime resistant isolates were Serratia spp., which are inherently cefuroxime resistant.

For gram-positive organisms, fluoroquinolones also appear a reasonable choice, particularly for $S$. pneumoniae infections. However, $9 \%$ of $S$. aureus strains were resistance to ciprofloxacin compared with $0 \%$ resistance to gentamicin in our study. Previous reports suggest there to have been an increase in the resistance in $S$. aureus and Streptococcus to ciprofloxacin and ofloxacin $(22 \%$ and $18 \%$, respectively) [19]. Our gram-positive resistance rate to fluoroquinolones was lower than that seen in other reports $[4,19]$. The suggestion from these studies is that although, fluoroquinolones are a reasonable first-line choice for grampositive bacteria, in cases slow to respond it is appropriate to switch to dual therapy early [4].

Gentamicin and cefuroxime are used as second-line dual treatment. Gentamicin, however, is considered to be toxic to the ocular surface and the difficulty to administer dual therapy may act as a limitation. Although our data set does not have complete information regarding cefuroxime response, it appears that fluoroquinolone monotherapy provides equal sensitivity as gentamicin therapy for most of the organisms studied (with $S$. aureus as an exception), therefore levofloxacin monotherapy remains a suitable first-line treatment. The use of monotherapy is also supported by a randomised control trial comparing the efficacy of monotherapy with fluoroquinolone to dual therapy with cefazolin and tobramycin [20]. Monotherapy was found to be equally efficacious but with fewer side effects [20]. The reasonable sensitivity to fluoroquinolone monotherapy seen in our study, is also similar to recent reports from other UK units $[3,4]$.

Limitations of this study include its retrospective nature as well as the lack of clinical information in the data set used. In practice, information such as prior antibiotic use, contact lens wear and clinical appearance is crucial when reviewing the corneal scrape result. It is also useful to determine the clinical significance of the cultured organism, particularly in the case of organisms forming part of the natural skin flora, such as CoNS. Thus, without clinical information the corneal scrape result alone needs to be analysed with caution. This is also true when considering AK cases. In clinical practice, patients have confocal microscopy, corneal scrapes sent for PCR to a reference laboratory and culture to the local laboratory. As outlined in a recent study, it is the combination of the three of these methods which is optimal in the diagnosis of AK [21]. Our data only included acanthamoeba species isolated by culture and not by PCR or confocal microscopy. The identification was by culture and therefore there is no speciation of the isolates. Clinically there were greater numbers of AK during the study period in these units, than that identified by this study alone (unpublished data).

In conclusion, this is the first study to report the microbial keratitis trends from the South West of England. Similar to that identified in other reports from the UK gram-positive bacteria were the most commonly identified group of organisms. In this study, there were no statistically significant trends in the identified organisms with time. Virulent organisms (e.g., $P$. aeruginosa, the Enterobacteriaceae, $S$. aureus and $S$. pneumoniae) were more likely to be detected on direct culture, compared with low-virulence organisms, such as CoNS, which were more likely to be detected on enrichment alone. There was good sensitivity to the antibiotics used in clinical practice suggesting that initial treatment with a topical fluoroquinolone as monotherapy appears to be a reasonable approach, with further treatment guided by the microbiological sensitivities for non-responsive cases.

\section{Summary}

\section{What was known before}

- Previous reports from the United Kingdom have suggested there to be trends in organisms responsible for microbial keratitis with time.

\section{What this study adds}

- There were no statistically significant trends in the identified organisms by corneal scrape, using BHI broth, with time over an 11-year study period in the South West of England. Virulent organisms (e.g., P. aeruginosa, the Enterobacteriaceae, S. aureus and S. pneumoniae) were more likely to be detected on direct culture, compared with low virulence organisms, such as CoNS, which were more likely to be detected on enrichment alone. There was good sensitivity to the antibiotics used in clinical practice, suggesting that initial treatment with a topical fluoroquinolone as monotherapy appears to be a reasonable approach, with further treatment guided by the microbiological sensitivities for non-responsive cases.

Acknowledgements Scottish Parasite Diagnostic Reference Section, Scottish Microbiology Reference Laboratories, Glasgow for acanthamoeba PCR. Moorfields Eye Hospital for confocal microscopy of acanthamoeba suspects

\section{Compliance with ethical standards}

Conflict of interest The authors declare that they have no conflict of interest. 
Publisher's note: Springer Nature remains neutral with regard to jurisdictional claims in published maps and institutional affiliations.

\section{References}

1. Whitcher JP, Srinivasan M, Upadhvav MP. Corneal blindness: a global perspective. Bull World Health Organ. 2001;79:214-21.

2. Allan BD, Dart JK. Strategies for the management of microbial keratitis. Br J Ophthalmol. 1995;79:777-86.

3. Ting DSJ, Settle C, Morgan SJ, Baylis O, Ghosh S. A 10-year analysis of microbiological profiles of microbial keratitis: the North East England Study. Eye. 2018;32:1416-7.

4. Tan SZ, Walkden A, Au L, Fullwood C, Hamilton A, Qamruddin A, et al. Twelve-year analysis of microbial keratitis trends at a UK tertiary hospital. Eye. 2017;31:1229-36.

5. Orlans HO, Hornby SJ, Bowler ICJW. In vitro antibiotic susceptibility patterns of bacterial keratitis isolates in Oxford, UK: a 10-year review. Eye. 2011;25:489-93.

6. Kaye SB, Rao PG, Smith G, Scott JA, Hoyles S, Morton CE, et al. Simplifying collection of corneal specimens in cases of suspected bacterial keratitis. J Clin Microbiol. 2003;41:3192-7.

7. Jasim H, Knox-Cartwright N, Cook S, Tole D. Increase in acanthamoeba keratitis may be associated with use of multipurpose contact lens solution. BMJ. 2012;344:e1246.

8. Kaye $S$, Tuft $S$, Neal T, Tole D, Leeming J, Figueiredo F, et al. Bacterial susceptibility to topical antimicrobials and clinical outcome in bacterial keratitis. Invest Ophthalmol Vis Sci. 2010;51:362-8.

9. Gopinathan U, Sharma S, Garg P, Rao GN. Review of epidemiological features, microbiological diagnosis and treatment outcome of microbial keratitis: experience over a decade. Indian $\mathbf{J}$ Ophthalmol. 2009;57:273-9.

10. Al-Dhaheri HS, Al-Tamimi MD, Khandekar RB, Khan M, Stone DU. Ocular pathogens and antibiotic sensitivity in bacterial keratitis isolated at King Khaled Eye Specialist Hospital, 2011 to 2014. Cornea. 2016;35:789-4.
11. Ferreira CS, Figueira L, Moreira-Gonçalves N, Moreira R, Torrão L, Falcão-Reis F. Clinical and microbiological profile of bacterial microbial keratitis in a portuguese tertiary referral center-where are we in 2015? Eye Contact Lens. 2018;44:15-20.

12. Hsiao CH, Sun CC, Yeh LK, Ma DH, Chen PY, Lin HC, et al. Shifting trends in bacterial keratitis in Taiwan: a 10-year review in a tertiary-care hospital. Cornea. 2016;35:313-7.

13. Zhang C, Liang Y, Deng S, Wang Z, Li R, Sun X. Distribution of bacterial keratitis and emerging resistance to antibiotics in China from 2001 to 2004. Clin Ophthalmol. 2008;2:575-9.

14. Alexandrakis G, Alfonso EC, Miller D. Shifting trends in bacterial keratitis in south Florida and emerging resistance to fluoroquinolones. Ophthalmology. 2000;107:1497-1502.

15. Shah A, Sachdev A, Coggon D, Hossain P. Geographic variations in microbial keratitis: an analysis of the peer-reviewed literature. Br J Ophthalmol. 2011;95:762-7.

16. Wilhelmus KR, Abshire RL, Schlech BA. Influence of fluoroquinolone susceptibility on the therapeutic response of fluoroquinolone-treated bacterial keratitis. Arch Ophthalmol. 2003;121:1229-33.

17. Kaye SB. Microbial keratitis and the selection of topical antibiotics. BMJ Open Ophthalmol. 2017;1:e000086.

18. Tavassoli S, Gunn D, O.Williams M, Darcy K. The successful treatment of a multidrug-resistant Achromobacter xylosoxidans corneal ulcer with topical meropenem. BMJ Case Rep. 2018; pii: bcr-2018-225163.

19. Goldstein MH, Kowalski RP, Gordon YJ. Emerging fluoroquinolone resistance in bacterial keratitis: a 5-year review. Ophthalmology. 1999;106:1313-18.

20. O'Brien TP, Maguire MG, Fink NE, Alfonso E, McDonnell P. Efficacy of ofloxacin vs cefazolin and tobramycin in the therapy for bacterial keratitis. Report from the Bacterial keratitis study research group. Arch Ophthalmol. 1995;113:1257-65.

21. JWY Goh, Harrison R, Hau S, Alexander CL, Tole DM, Avadhanam VS. Comparison of in vivo confocal microscopy, PCR and culture of corneal scrapes in the diagnosis of acanthamoeba keratitis. Cornea. 2018;37:480-5. 